\title{
Thickness-controlled Three-dimensional Dirac Semimetal for Scalable High-performance Terahertz Optoelectronics
}

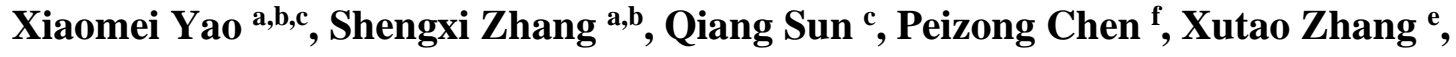

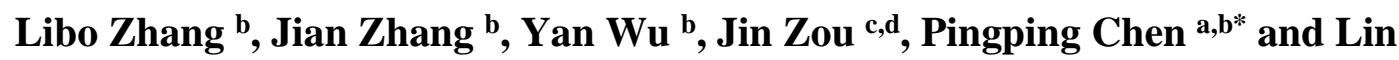 \\ Wang ${ }^{\text {a,b** }}$
}

\section{Supporting Information:}

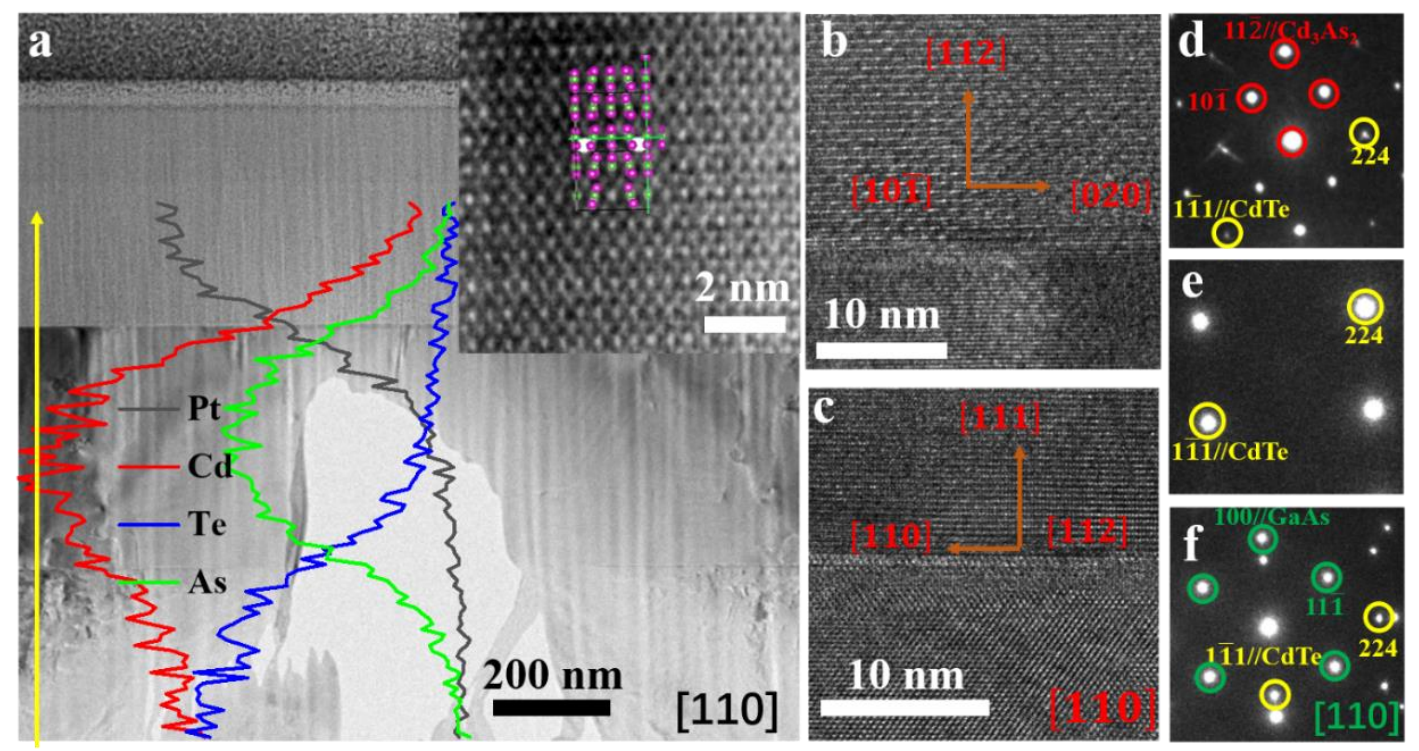

Figure S1: Microstructure of the film with the thickness of $400 \mathrm{~nm}$. (a): Overall view of sample fabricated by FIB. The inset is the EDS result of the cross-section sample along the yellow arrow. Inset in the right side is the amplified HRTEM image, indicating the regular crystal structure of $\mathrm{Cd}_{3} \mathrm{As}_{2}$. (b): HRTEM result of $\mathrm{Cd}_{3} \mathrm{As}_{2} / \mathrm{CdTe}$ interface. (c): HRTEM result of $\mathrm{CdTe} / \mathrm{GaAs}$ interface. (d): SAED result of $\mathrm{Cd}_{3} \mathrm{As}_{2} / \mathrm{CdTe}$ interface. (e): SAED result of CdTe. (f): SAED result of $\mathrm{CdTe} / \mathrm{GaAs}$ interface.

Fig. S1 shows the morphology characteristics of the $400 \mathrm{~nm}$ film. Fig. S1(a) is the cross-section sample fabricated by FIB (Focused Ion Beam). The inset is the line-scan 
EDS results along the yellow arrow, indicating two well-defined interfaces: $\mathrm{Cd}_{3} \mathrm{As}_{2} / \mathrm{CdTe}$ interface and $\mathrm{CdTe} / \mathrm{GaAs}$ interface. In the right side, the amplified HRTEM image of $\mathrm{Cd}_{3} \mathrm{As}_{2}$ film demonstrates the regular crystal structure and high crystal quality. The HRTEM images of the two interfaces are shown in Fig. S1(b) and (c), and the corresponding SAED results are shown in Fig. S1(d)-(f). The thickness of the film is about $400 \mathrm{~nm}$ and the atomic ratio between $\mathrm{Cd}$ and As is about 3:2. In CdTe layer, the atomic ratio is 1:1. From the SAED results, we can distinguish two sets of diffraction patterns in the $\mathrm{Cd}_{3} \mathrm{As}_{2} / \mathrm{CdTe}$ interface, $\mathrm{Cd}_{3} \mathrm{As}_{2}$ and $\mathrm{CdTe}$, respectively. Similarly, in $\mathrm{CdTe} / \mathrm{GaAs}$ interface, CdTe and GaAs diffraction spots can be demonstrated in Fig. S1(f). Pure CdTe diffraction pattern is shown in Fig. S2(e). The calculated value of the space distance based on the SAED pattern is in coincidence with the XRD results. The epitaxial relation between $\mathrm{CdTe}$ and $\mathrm{Cd}_{3} \mathrm{As}_{2}$ can be concluded from Fig. S1 as well. The calculated lattice mismatch between $\mathrm{CdTe}$ and $\mathrm{Cd}_{3} \mathrm{As}_{2}$ is $2.4 \%$, while the value between GaAs and CdTe is $1.5 \%$. 

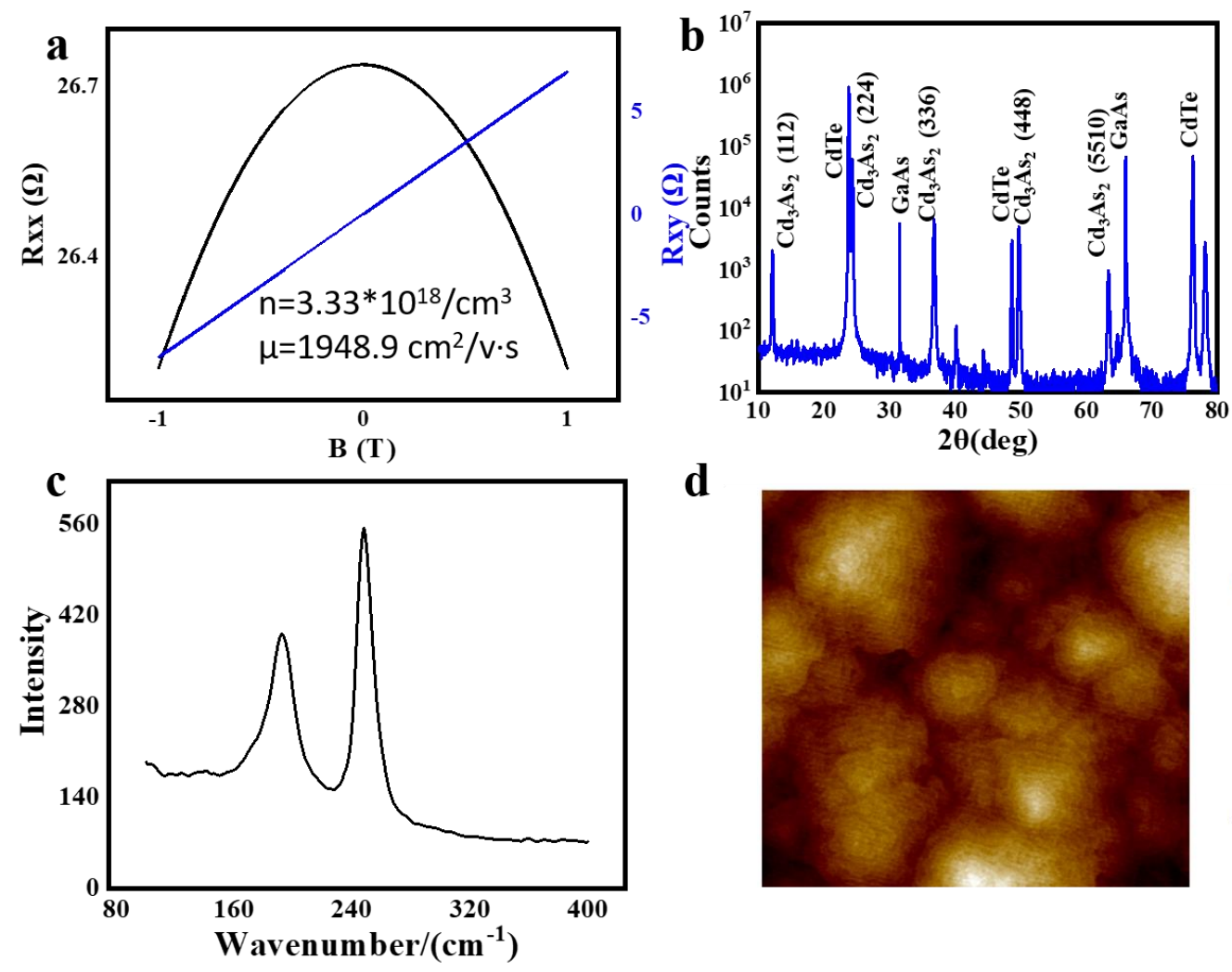

d

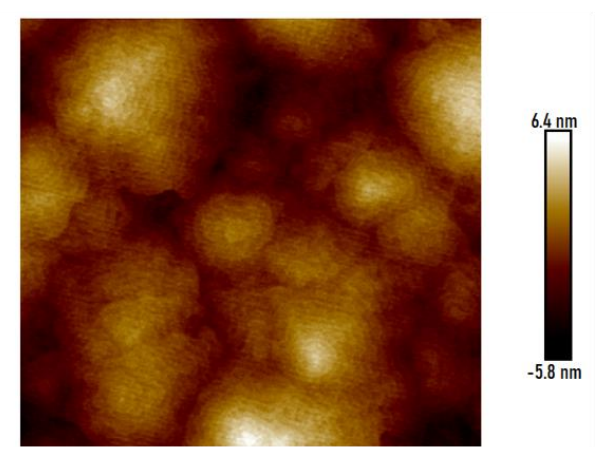

Figure S2: Fundamental properties of $\mathrm{Cd}_{3} \mathrm{As}_{2}$ film with the thickness of $400 \mathrm{~nm}$. (a) Hall measurement result with the bias current set on $1 \mathrm{~mA}$. (b) Full range XRD pattern demonstrating the growth direction of CdTe buffer layer and $\mathrm{Cd}_{3} \mathrm{As}_{2}$ layer. (c) Raman result of the film. (d) AFM image of the film surface.

Fig. S2 shows the fundamental physical characters of the MBE grown $\mathrm{Cd}_{3} \mathrm{As}_{2}$ film with thickness of $400 \mathrm{~nm}$. The electron mobility and concentration of the as-grown epilayer are $1948.9 \mathrm{~cm}^{2} / \mathrm{V} \cdot \mathrm{s}$ and $3.33 \times 10^{18} / \mathrm{cm}^{-3}$, which are obtained by the Vanderbilt method shown in Fig. S2(a). Full range XRD pattern shown in Fig. S2(b) with the peaks matched to the lattice constants of a tetragonal body center crystal structure ( $\mathrm{P} 4_{2} / \mathrm{nmc}$ (137)) as $\mathrm{a}=\mathrm{b}=7.4 \AA$ and $\mathrm{c}=9.3 \AA$. The peaks of $\mathrm{CdTe}(111)$ and $\mathrm{Cd}_{3} \mathrm{As}_{2}(112)$ are wellfitted to the reference peaks using a Voigt function. Raman spectra were recorded at room temperature from a spectrometer equipped with a $514.5 \mathrm{~nm}$ laser, revealing two 
peaks of $A=191.2 \mathrm{~cm}^{-1}$ and $\mathrm{B}=245.7 \mathrm{~cm}^{-1}$ clearly as shown in Fig. S2(c). The AFM result shown in Fig. S2(d) demonstrates a rather smooth film with the root mean square roughness (RMS) about $1.6 \mathrm{~nm}$ and fluctuation smaller than $10 \mathrm{~nm}$. On the surface, island-like clusters about $10 \mathrm{~nm}$ appear, indicating a layered step growth mode.
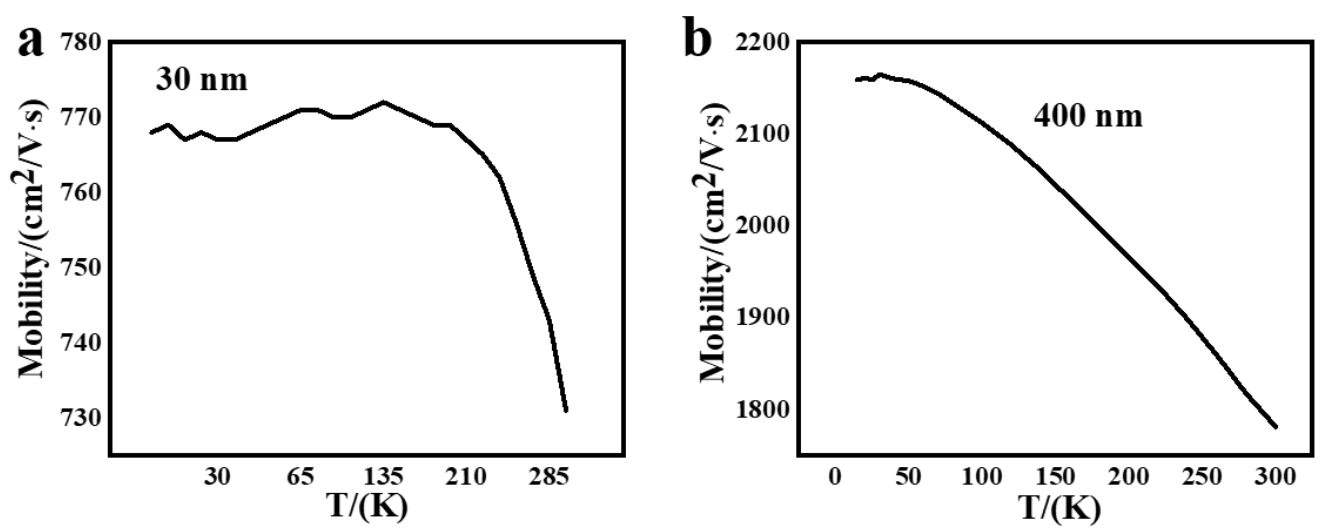

Figure S3: Temperature-dependent mobility of films with thickness of $30 \mathrm{~nm}$ and $400 \mathrm{~nm}$, respectively.

The values of mobility with varied temperature of the two samples are shown in Fig. S3. Overall, mobility of sample with thickness of $30 \mathrm{~nm}$ (a) is smaller than the sample with $400 \mathrm{~nm}$ thickness (b). With the reduction of film thickness, enhanced scattering from interface imperfections for thinner films affect the mobility of the film severely. Worth to note that, the increased temperature would also give the mobility an obvious decrease over all measurement temperature range. 


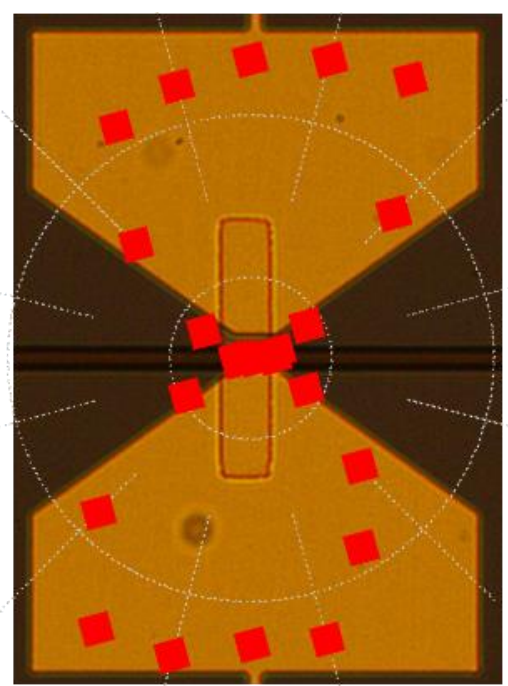

Figure S4: Optical microscope image of the obtained device and electromagnetic-polarization varied performance.

Fig. S4 is the optical microscope image of the obtained device and electromagneticpolarization varied performance, indicating the strong polarization dependence of photocurrent and the response can be improved by an order of magnitude when the $\mathrm{THz}$ electric-field is polarized along the direction of antenna arms, substantiating the importance of electromagnetic coupling process arrow. 


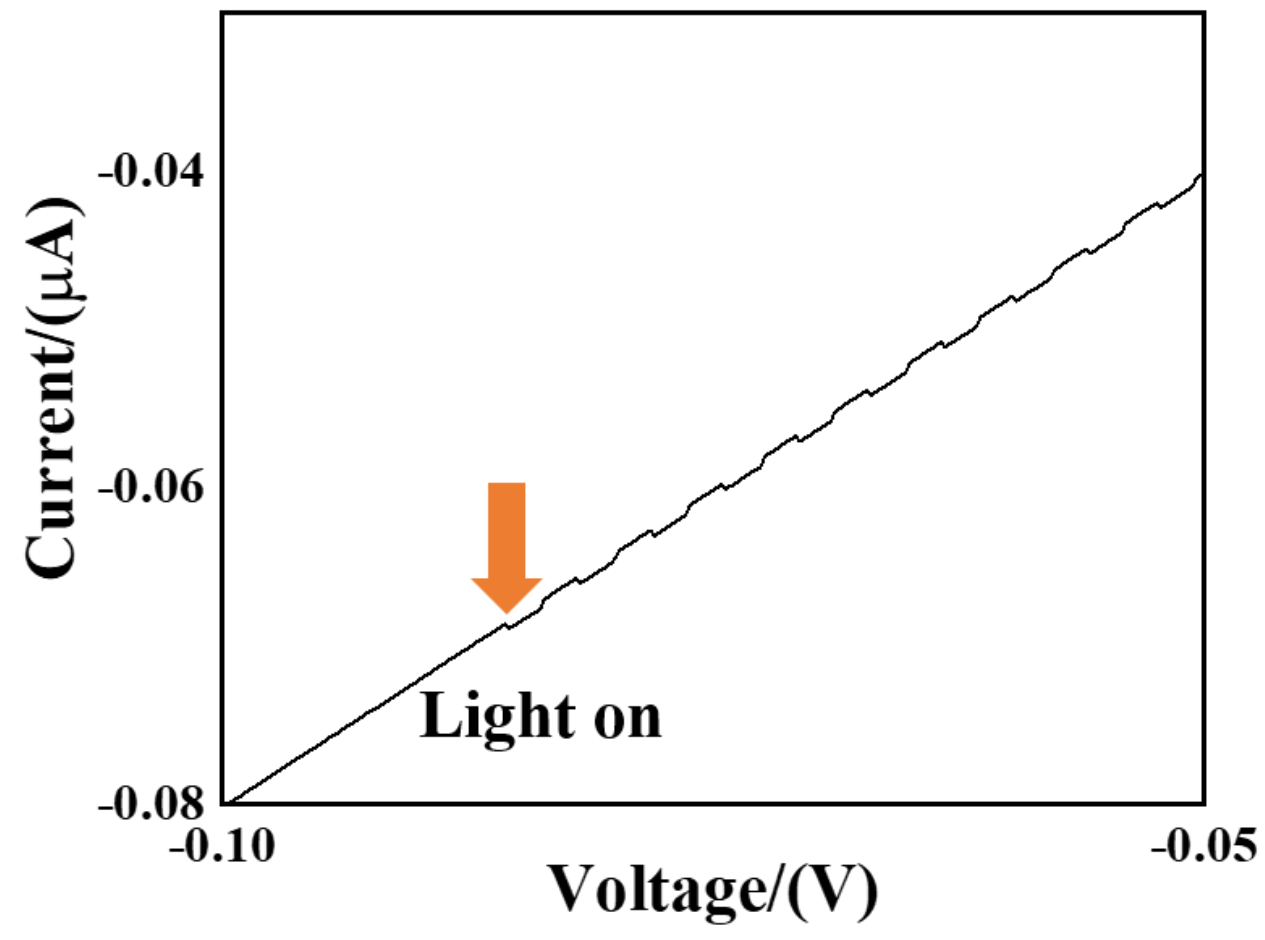

Figure S5: IV curve of the device shown in Fig. 4 (g), and the THz radiation is turned on at the yellow arrow.

Fig. S5 is the supplementary evidence of the PTE effect in the asymmetry device shown in Fig. 4(g). The IV-curve shown is obtained at a negative bias voltage, indicating the current direction is from the ground to the opening end, while the direction of electrons is reverse. The current increases with the $\mathrm{THz}$ radiation turned on which demonstrate the direction of extra electrons is in consistence with the dark current. Hence, the direction of extra electrons could be demonstrated as from opening end to the ground. 